\title{
Feasibility of interstitial stepping-source electronic brachytherapy to locally inoperable tumors
}

\author{
Arne Mathias Ruder, MD*, Laurens Inghelram*, Frank Schneider, PhDl, Gustavo R. Sarria, MD', Jürgen Hesser, PhD², \\ Frederic Bludau, MD³, Udo Obertacke, MD³, Frederik Wenz, MD',4, Yasser Abo-Madyan, MD', Frank A. Giordano, MD5 \\ 'Department of Radiation Oncology, University Medical Center Mannheim, Mannheim Medical Faculty, Heidelberg University, Germany, \\ ${ }^{2}$ Department of Data Analysis and Modeling, Mannheim Institute for Intelligent Systems in Medicine, University Medical Center Mannheim, \\ Mannheim Medical Faculty, Heidelberg University, Germany and Interdisciplinary Center for Scientific Computing (IWR), Heidelberg University. \\ Germany and Central Institute for Computer Engineering (ZITI), Heidelberg University, Germany, ${ }^{3}$ Department of Orthopedic and Trauma \\ Surgery, University Medical Center Mannheim, Mannheim Medical Faculty, Heidelberg University, Germany, ${ }^{4}$ University Medical Center \\ Freiburg, Freiburg, Germany, ${ }^{5}$ Department of Radiation Oncology, University Hospital Bonn, University of Bonn, Germany \\ *Arne Mathias Ruder and Laurens Inghelram contributed equally to this work.
}

\begin{abstract}
Purpose: Radiotherapy is the mainstay in the treatment of locally inoperable tumors. Interstitial electronic needlebased kilovoltage brachytherapy (EBT) could be an economic alternative to high-dose-rate (HDR) brachytherapy or permanent seed implantation (PSI). In this work, we evaluated if locally inoperable tumors treated with PSI at our institution may be suitable for EBT.

Material and methods: A total of 10 post-interventional computed tomography (CT) scans of patients, who received PSI and simulated stepping-source EBT applied with Intrabeam system and needle applicator were used. EBT treatment planning software with 3-dimensional image and projection of applicator were applied for designing trajectories and establishing dwell positions. Dwell position doses were summarized, and doses covering $90 \%$ of the target volume $\left(\mathrm{D}_{90}\right)$ achieved with stepping-source EBT were compared to those of PSI. Additionally, conformality of dose distributions and total irradiation time were assessed using conformation number $(\mathrm{CN})$ or conformal index (COIN).

Results: In all patients, $\mathrm{D}_{90}$ of EBT exceeded the prescribed dose or $\mathrm{D}_{90}$ of PSI on average by $4.7 \%$ or $21.3 \%$ relative to the prescribed dose, respectively. Mean number of trajectories was 5.0 for EBT and 6.9 for PSI. Average CN/COIN for EBT was 0.69 , with a mean irradiation time of 27.8 minutes for standardized dose of 13 Gy.

Conclusions: Stepping-source EBT allowed for a conformal treatment of inoperable interstitial tumors with similar $\mathrm{D}_{90}$. Fewer trajectories were required for EBT in majority of cases.

Key words: electronic brachytherapy, interstitial brachytherapy, iodine seeds, Intrabeam, stepping source.

\section{Purpose}

Inoperable primary or secondary tumors (metastases) are frequently treated with interstitial brachytherapy, which allows for application of high radiation doses to a lesion, with a steep dose fall-off outside of the target and sparing surrounding (healthy) tissue [1,2,3,4]. Multiple brachytherapy treatment modalities are available, including catheter-based high-dose-rate (HDR) afterloading brachytherapy with ${ }^{192}$ Ir sources or permanent implantation with ${ }^{125}$ I seeds (PSI), as the most used methods [5].

An emerging technical alternative may be electronic brachytherapy (EBT) with miniaturized linear accelerators that generate low-energy $\mathrm{X}$-rays, which are rapidly absorbed in surrounding tissue, showing dose gradients similar to PSI and steeper than in HDR [6]. In contrast to HDR and PSI, EBT does not require radionuclides, source replacements, or post-interventional precautions. Additionally, EBT devices are mobile and may be used in any room that fulfils basic requirements for operating kV-energy emitting X-ray devices (e.g., classical X-ray imaging, C-arm fluoroscopy, or computed tomography CT) $[7,8,9]$. Current applications of EBT mostly involve intracavitary (intraoperative) radiotherapy of breast, brain, and spine tumors, but also intravaginal or intrauterine EBT and superficial EBT of skin tumors [10,11, $12,13,14,15,16]$.

To evaluate whether EBT is a feasible alternative to contemporary standard technology, we created radiation treatment plans with stepping source EBT in post-im-
Address for correspondence: Arne Mathias Ruder, MD, Department of Radiation Oncology, University Medical Center Mannheim, Theodor-Kutzer-Ufer 1-3, 68163 Mannheim, Germany, phone: +49 621 383 6020, fax: +49 621 383 3439, e-mail: arne.ruder@umm.de
Received: 24.02 .2020

Accepted: 16.07 .2020

Published: 30.10 .2020 
plantation CT scans of patients treated with PSI, and assessed the conformality, doses covering $90 \%$ of the target volume $\left(\mathrm{D}_{90}\right)$, and required trajectories in comparison to PSI as well as estimated treatment times for EBT.

\section{Material and methods}

\section{Data set}

Post-implantation CT data sets (DICOM files; Brillance CT Big Bore, Koninklijke Philips N.V., Amsterdam, The Netherlands; resolution: $512 \times 512$, field of view: $600 \mathrm{~mm}$ $\times 600 \mathrm{~mm}$, slice thickness: $1 \mathrm{~mm})$ of 10 patients $(n=10)$ that received CT-guided PSI $\left({ }^{125} \mathrm{I}\right.$ seeds, Eckert \& Ziegler BEBIG GmbH, Berlin, Germany; activity at implantation between $0.43 \mathrm{mCi}$ and $0.66 \mathrm{mCi}$, IPSA planning based on the TG-43 formalism with Oncentra prostate versions 4.0.7-4.2.2.4, Elekta AB, Stockholm, Sweden) alone or as a boost in combination with external beam radiotherapy (EBRT) to inoperable tumors progressing on standard therapy were chosen as templates for EBT treatment planning. This cohort represented all patients treated with CT-guided PSI for inoperable interstitial tumors from 2012 to 2019 in our department. All analyses were performed after approval of the institutional review board.

\section{Target volumes and dose prescription}

If a patient had received therapy with PSI only, the same planning target volume (PTV) was used for the simulation of EBT. If PSI had been combined with EBRT, the intent was either to boost the whole tumor or to treat parts of the tumor before EBRT (e.g., when parts of the tumor were in close proximity to organs at risk [OARs]). In case of partial treatment with PSI, the PTV still resembled the whole tumor and no dedicated partial PTV was done to treat as much of the PTV as possible with PSI. To estab- lish a realistic partial PTV for simulation of EBT ex post, the $100 \%$-isodose line of PSI was transformed into a structure in Velocity 3.2.1 (Varian Medical Systems, Palo Alto, CA, USA), and was considered as PTV. Subsequently, this partial PTV from PSI represented the PTV for EBT. For relative comparison only, previously prescribed PSI doses were adopted for EBT and required to cover $90 \%$ of the PTV for EBT.

\section{EBT system}

Electronic brachytherapy treatment was simulated using the Intrabeam system (Carl Zeiss Meditec AG, Oberkochen, Germany), with a needle-shaped applicator, which allowed for a spherical delivery of low-energy X-rays (maximum energy, $50 \mathrm{kV}$ ) emitted from the tip of applicator with a steep dose gradient (Figure 1) [17].

\section{EBT treatment planning}

Computed tomography studies and structure files (PTVs, OARs) were imported into Radiance 4.0.3/4.0.5 (GMV SA, Madrid, Spain). First, interstitial trajectories were chosen according to all requirements of a realistic needle placement (avoidance of organs, major vessels, nerves, and bones). Then, dwell positions were defined in $10 \mathrm{~mm}$ increments along the defined trajectories. Next, dose distributions were calculated multiple times for every dwell position, each time with differing prescription radii, using a non-TG-43 model-based dose calculation in a hybrid form of Monte Carlo-based algorithm for the distribution of $\mathrm{kV}$-photons in heterogeneous tissue $[18,19]$. For dwell position dose summation, the CT studies and multiple dose files for each dwell position were exported to Velocity. By manual forward planning, a combination of dwell position doses was selected to generate a sum dose with the most conformal coverage of the PTV by the $\mathrm{D}_{90}$
A

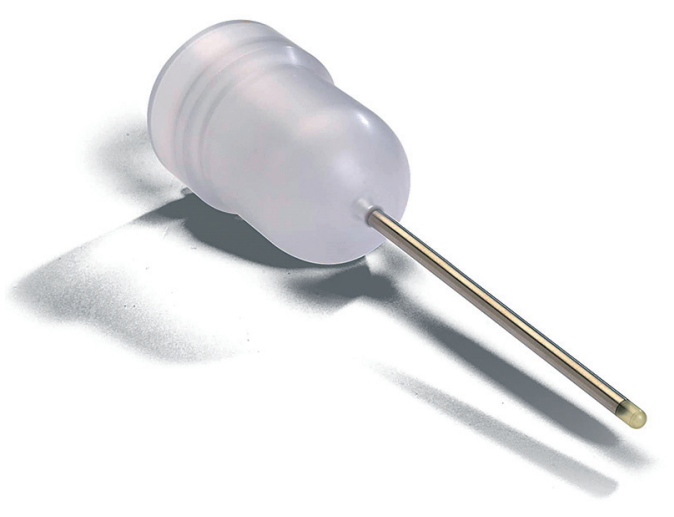

B

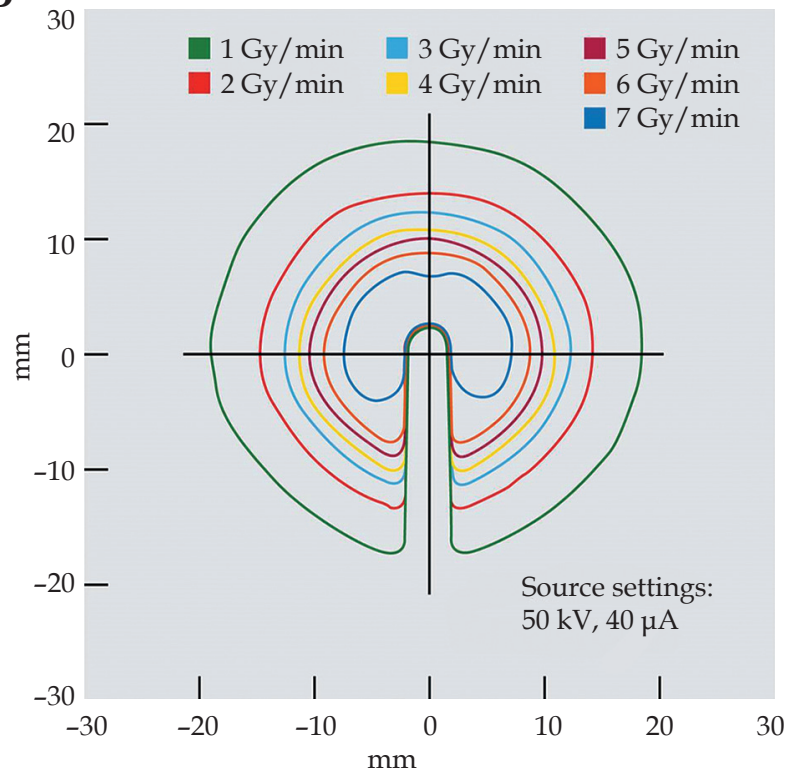

Fig. 1. Applicator and dose rates. A) The needle applicator used for this planning study, and B) depicts the corresponding dose rates at defined distances from the tip 
of prescription dose stemming from PSI. To obtain conformality, the refinement of dwell positions along a trajectory was performed by increasing or decreasing the distance between dwell positions. Subsequently, dwell position dose calculation and forward planning were recommenced.

\section{Plan evaluation}

For each case, the sum dose of EBT to $90 \%$ of the PTV $\left(\mathrm{D}_{90}\right.$ of EBT) was compared to the prescribed dose of EBT (adopted from PSI) and to originally reached $\mathrm{D}_{90}$ by PSI. If the $\mathrm{D}_{90}$ of EBT met the prescribed dose stemming from PSI, or if the $\mathrm{D}_{90}$ of EBT was higher than the $\mathrm{D}_{90}$ achieved with PSI, conformality was evaluated. Prior to conformality assessment, virtual dose distribution was corrected if dose accumulation in disruptive elements outside of the PTV occurred, including urethral catheters or metal implants as well as dose distribution outside the patient (dose to air). By determining the size of PTV, the volume of tissue enclosed by the isodose line of prescribed dose and the volume of PTV covered by the prescribed dose $\left(\mathrm{V}_{100}\right)$, the conformation number $(\mathrm{CN})$ was calculated [20]. If OARs were affected by the isodose line of prescribed dose, conformality was assessed with the conformal index (COIN) by multiplying the $\mathrm{CN}$ with an additional term, representing the fractions of OARs receiving the prescribed dose or more [21]. Only plans with values of CN or COIN greater than 0.6 were accepted as conformal [20]. If the $\mathrm{D}_{90}$ of EBT was lower than the prescribed dose or the $\mathrm{D}_{90}$ of PSI, or if conformality was not reached, the planning was repeated with alterations to dwell positions.

\section{Irradiation time}

For the estimation of beam-on time during the treatment, all acquired conformal sum doses were virtually standardized to 13 Gy by adjusting the dwell position doses in relation to each other. Here, 13 Gy represents a single-fraction dose for interstitial brachytherapy, corresponding to an equivalent dose in 2 Gy fractions $\left(\mathrm{EQD}_{2}=n \times D \times((d+\mathrm{\alpha} / \beta) /(2+\mathrm{\alpha} / \beta))\right)$ between 32.5 and $54 \mathrm{~Gy}$ (for an $\alpha / \beta$-ratio between 3 and 10 ), considering the increased relative biological effectiveness (RBE) of 1.3 for low-energy $\mathrm{kV}$-photons [2,22,23,24].

\section{Results \\ Patients' characteristics}

This planning study was conducted using CT scans of 10 cases, of which 5 patients obtained PSI to treat local (or loco-regional) tumor recurrence, and 5 patients received PSI to palliate symptoms of metastases. Six of the patients were females and 4 males, and the average age at PSI treatment was 62.5 years (standard deviation $[S D]=11.6)$. The entities of tumors and further details are presented in Table 1.

\section{Planning}

Doses were adopted from PSI and prescribed to $90 \%$ of the PTV for EBT, with a minimum of $10 \mathrm{~Gy}$, a maximum of $60 \mathrm{~Gy}$, and an average of $31 \mathrm{~Gy}(\mathrm{SD}=14.6)$. The average volume of PTV was $50.6 \mathrm{~cm}^{3}$ (SD = 34.5), with a minimum of $11.9 \mathrm{~cm}^{3}$ and a maximum of $106.7 \mathrm{~cm}^{3}$. The total number of iodine seeds implanted during PSI ranged between 5 and 28, with an average of 15 iodine seeds $(\mathrm{SD}=7.6)$, which required between 4 and 11 needle trajectories (average, 6.9, SD = 2.2). For EBT, the total number of trajectories per case varied between 1 and 13, with an average of $5.0(S D=3.2)$. All trajectories for EBT fulfilled requirements of a realistic puncture. In comparison to PSI, the number of trajectories needed for EBT was lower in 7 cases and the same in 2 cases. In 1 case, ad-

Table 1. Patients' characteristics and results of simulated interstitial EBT treatment. Table consists of underlying diseases, locations of the tumors treated, planning target volume for electronic brachytherapy (PTV $\mathrm{EB}_{\mathrm{B}}$ ), number of trajectories and dwell points, conformation number (CN) and conformal index (COIN), and beamon time for a standardized dose of 13 Gy

\begin{tabular}{|c|c|c|c|c|c|c|c|}
\hline Patient & Primary & Location & $\begin{array}{l}\text { Volume of } \\
\text { PTV }_{E B}\left(\mathrm{~cm}^{3}\right) \\
\end{array}$ & $\begin{array}{c}\text { No. of } \\
\text { trajectories }\end{array}$ & $\begin{array}{l}\text { No. of dwell } \\
\text { points }\end{array}$ & $\mathrm{CN}^{\#}$ or $\mathrm{COIN}^{*}$ & $\begin{array}{l}\text { Beam-on time } \\
\text { for } 13 \mathrm{~Gy}(\mathrm{~min})\end{array}$ \\
\hline 1 & Anal carcinoma & Vulva & 20.5 & 2 & 3 & $0.74^{\#}$ & 16.8 \\
\hline 2 & Breast carcinoma & Sternum & 94.6 & 7 & 20 & $0.66^{*}$ & 42.0 \\
\hline 3 & Fibrosarcoma & Left shoulder & 16.6 & 3 & 5 & $0.70^{\#}$ & 8.9 \\
\hline 4 & Fibrosarcoma & Retroperitoneum & 106.7 & 5 & 8 & $0.63^{\star}$ & 53.0 \\
\hline 5 & Leiomyosarcoma & Left shoulder & 15.6 & 1 & 2 & $0.89^{\#}$ & 8.2 \\
\hline 6 & $\begin{array}{c}\text { Malignant mela- } \\
\text { noma }\end{array}$ & Lower back & 82.8 & 13 & 25 & $0.65^{\star}$ & 43.4 \\
\hline 7 & $\begin{array}{c}\text { Malignant mela- } \\
\text { noma }\end{array}$ & Pelvis & 67.2 & 3 & 7 & $0.69^{\#}$ & 36.8 \\
\hline 8 & Rectal carcinoma & Pelvis & 63.1 & 6 & 15 & $0.62^{*}$ & 31.8 \\
\hline 9 & Vulvar carcinoma & Pelvis & 27.0 & 6 & 13 & $0.65^{\#}$ & 29.5 \\
\hline 10 & Vulvar carcinoma & Pelvis & 11.9 & 4 & 11 & $0.67^{\#}$ & 8.2 \\
\hline
\end{tabular}


A
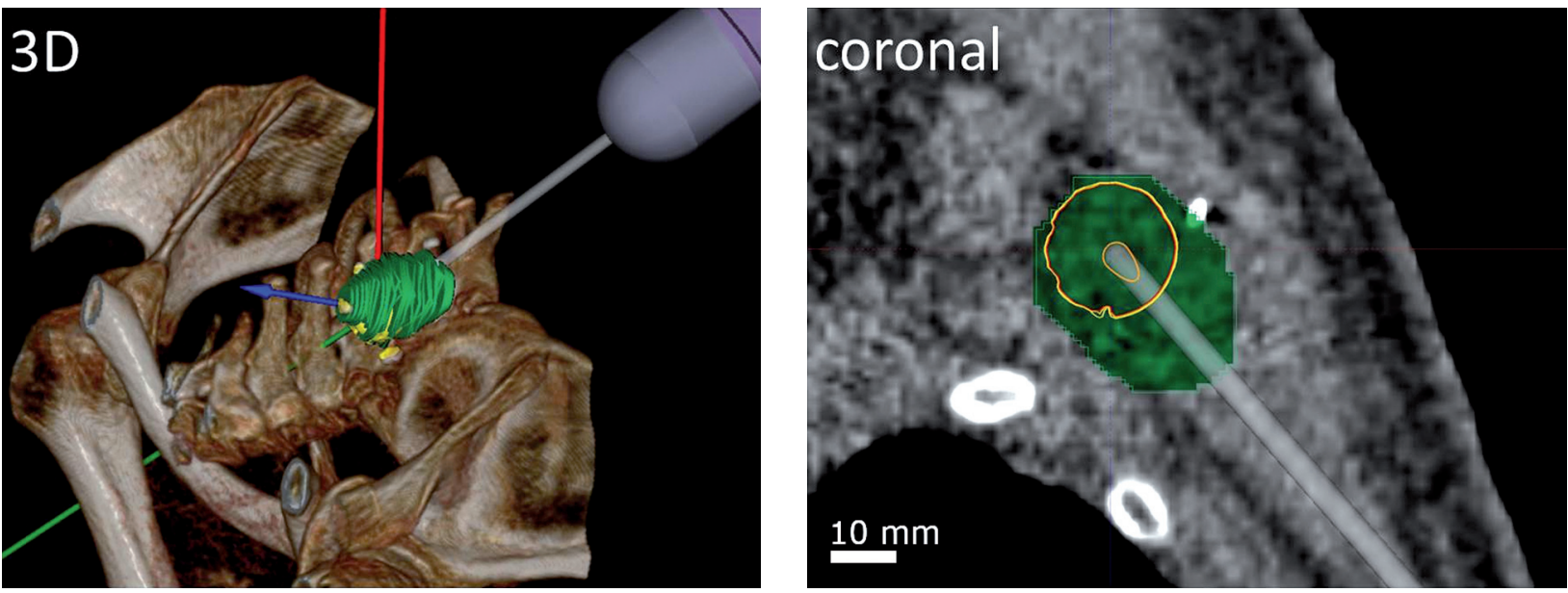

B
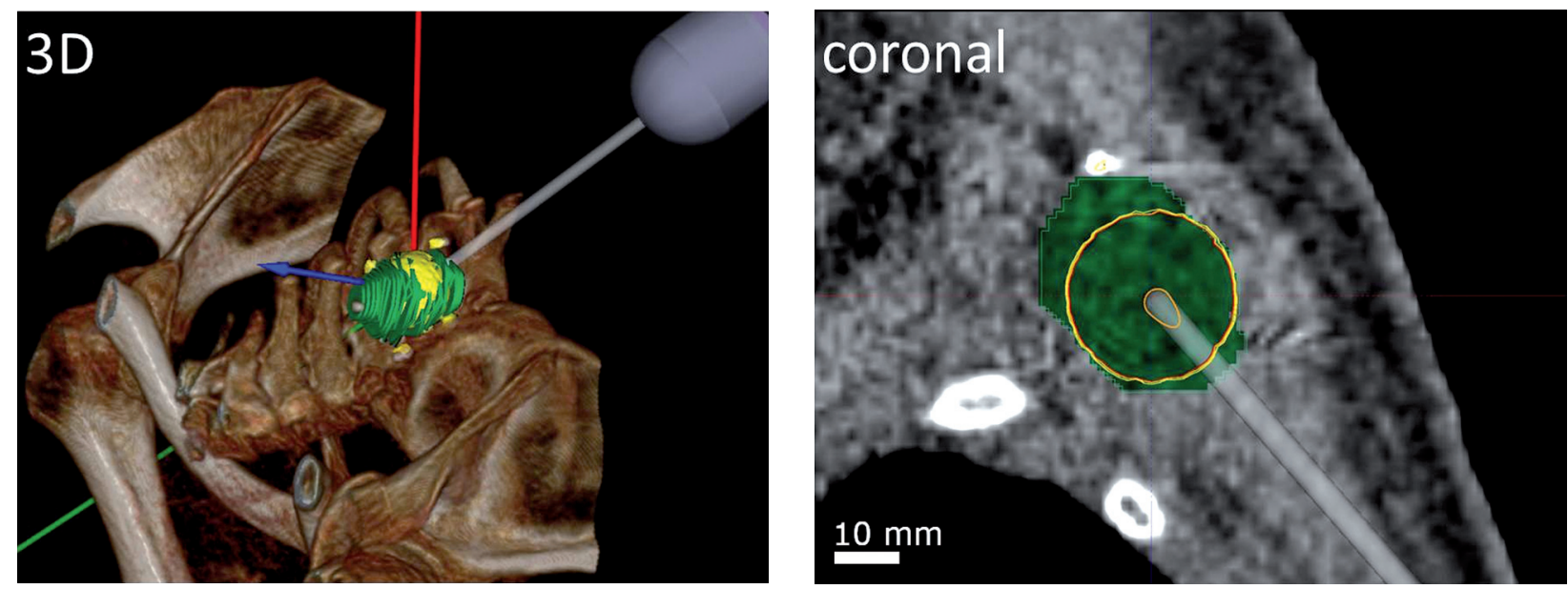

Fig. 2. Simulation of the needle applicator stepping along a virtual trajectory in a lesion in the left shoulder region. The PTV (green) contains two dwell positions, with (A) dwell position 1 and (B) dwell position 2, each displayed in 3-dimensionally rendered view (3D) and in a coronal plane. The dose distributions of dwell positions are displayed as isodose lines of 90\%, 95\%, and $100 \%$ of the prescribed dose in yellow, orange, and red, respectively

ditional trajectories were necessary for EBT. Overall significance for less trajectories in EBT could not be shown in this cohort (Mann-Whitney $U$ test, two-tailed, significance at $p \leq 0.05, U$-value $=25, Z$-score $=1.85203, p$-value $=0.06432)$. The number of utilized dwell positions for EBT ranged from 2 to 25 , with a mean of 10.9 (SD = 7.1) dwell positions per case. On average, $2.1(\mathrm{SD}=1.1)$ dwell positions per trajectory were generated, with a minimum of 1 and a maximum of 4 dwell positions. An example of the simulated needle applicator stepping along a trajectory is shown in Figure 2. On average, total forward planning time per case was $3.0 \mathrm{~h}(\mathrm{SD}=1.4)$, with a range from $0.8 \mathrm{~h}$ to $5.7 \mathrm{~h}$. Moreover, planning time strongly correlated with the number of trajectories and dwell positions (Pearson's correlation coefficient, $r(8)=0.94, p<0.05$, and $r(8)=0.98, p<0.05)$

\section{Coverage, conformality, and beam-on time}

For all cases of simulated EBT, either the prescribed dose or a dose greater than the $\mathrm{D}_{90}$ obtained by PSI was reached. In 8 cases, the $\mathrm{D}_{90}$ acquired with EBT met the prescribed dose, exceeding it by an average of $4.7 \%(\mathrm{SD}=3.9)$, with a maximum of $12.6 \%$. The prescribed $\mathrm{D}_{90}$ was not achieved in 2 cases, but still exceeded the $\mathrm{D}_{90}$ obtained with PSI (average, $21.3 \%, \mathrm{SD}=20.1$ ) relative to the prescribed dose. The $\mathrm{CN}$ or COIN, respectively, were greater than 0.6 in all 10 cases, with values between 0.62 and 0.89 , and an average of $0.69(\mathrm{SD}=0.075)$. Two examples of conformal dose coverage of the PTV $\mathrm{EBT}_{\mathrm{ET}}$ by EBT are shown in Figure 3. The mean beam-on treatment time for EBT with the standardized dose of 13 Gy was $27.8 \mathrm{~min}(\mathrm{SD}=15.6)$.

\section{Discussion}

Here, for the first time, we demonstrated that stepping-source EBT may pose as a technical alternative to PSI for interstitial treatment of inoperable tumors, offering conformality but requiring less trajectories in most of our cases. EBT consistently achieved CN or COIN index values $>0.6$, which is an accepted cut-off for a con- 
A
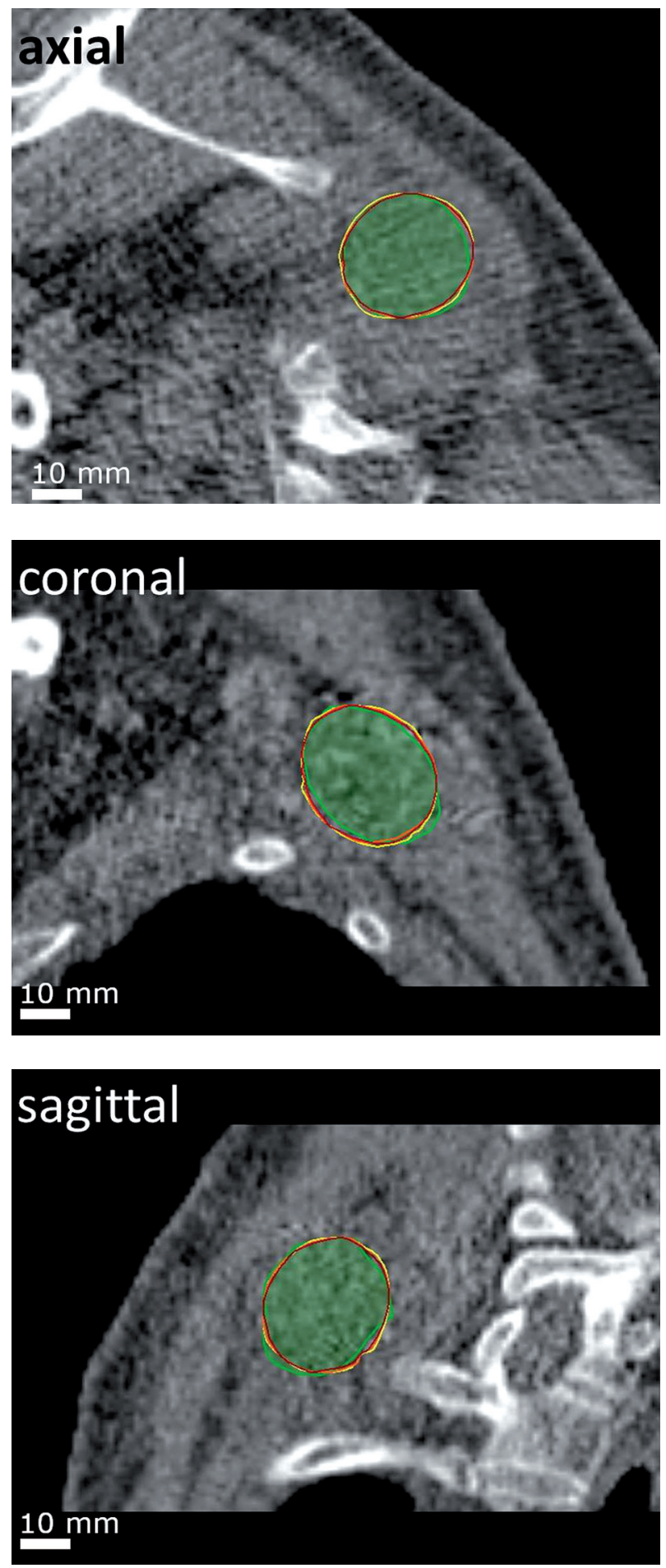

B
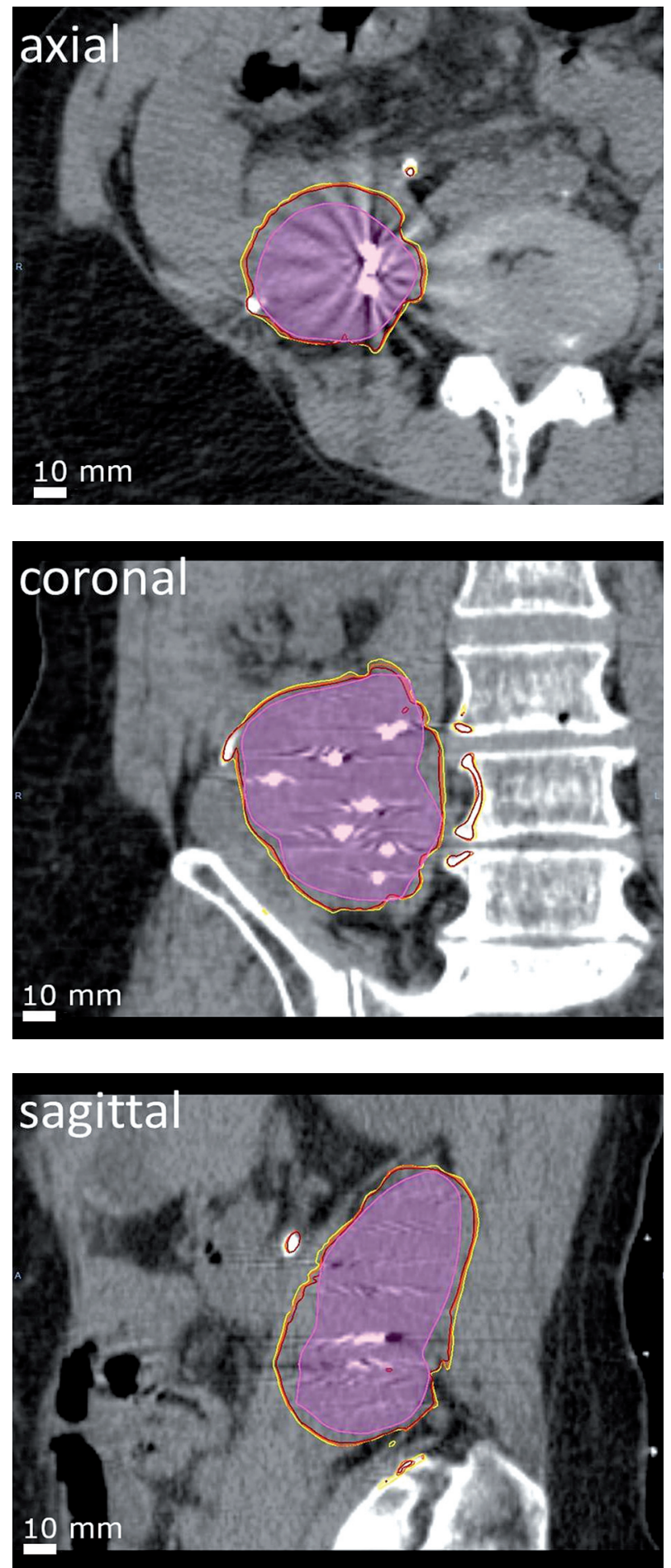

Fig. 3. Dose coverage after dwell position summation. Shown are two exemplary cases. A) For the first case (also displayed in Figure 2), a CN of 0.89 was achieved for a PTV in the left shoulder region (green). B) For the second case, a COIN of 0.63 was achieved for a PTV in the retroperitoneal space (pink). Isodose lines of $90 \%, 95 \%$, and $100 \%$ of the prescribed dose displayed in yellow, orange, and red, respectively

formal dose distribution [20,21]. Although PSI and EBT have fundamental differences in dose rate and biological effectiveness, the nominal doses of PSI for relative comparison and assessment were prescribed [23]. As for dose adoption, up- or downscaling of the sum dose after the completion of EBT forward planning is facile, since dose is a function time in EBT and dwell position doses can be adapted in relation to each other to reach a de- 
sired sum dose. In a clinical setting, the dose prescription for EBT need to be individualized, depending on the overall treatment intention (curative or palliative), $\alpha / \beta$-ratio of the tumor, and tolerance doses of surrounding tissues or organs. Moreover, the overall beam-on time mainly depends on the size of PTV, tissue density, and desired dose. When estimating the beam-on time with a standardized dose of $13 \mathrm{~Gy}$, we never exceeded 30 minutes. Presumably, the prescription dose would considerably be lower in cases with palliative intent or due to OARs constraint, resulting in even shorter beamon time.

A limitation of our study can be seen in the rigid registration of post-interventional CT scans, which did not allow for simulation of tissue deformation caused by needle applicator when calculating the dose distribution. Furthermore, these images also contained titaniumencapsulated seeds, which locally influence the dose distribution of EBT in the PTV. A particularity of EBT and iodine seeds is the absorption property of low-energy X-rays, with dose gradients of up to $50 \%$ per millimeter in close proximity to the applicator, provoking an inhomogeneity in the dose distribution with parts of the PTV receiving substantially more than $100 \%$ of the prescribed dose $[25,26]$. A greater number of dwell positions could mitigate these hot spots, but the treatment of lesions that contain radiation-sensitive structures should be cautiously evaluated. On the other hand, the sharp dose gradient of low-energy X-rays allows for effective sparing of tissues around the PTV. A special attention was paid to trajectory planning, as the Intrabeam system used for this EBT simulation requires a low number of trajectories due to diameter of needle applicator $(4.2 \mathrm{~mm})$ to avoid excessive tissue trauma, and limits the lesion depth with its needle length of $94 \mathrm{~mm}$.

The number of necessary trajectories, with acquired level of conformality and acceptable beam-on time would allow for a transfer of stepping-source EBT into a clinical workflow, ideally as a CT-guided intervention. For an efficient procedure, a software algorithm for inverse planning with trajectory and dwell position optimization, as available for HDR and PSI (e.g., IPSA and HIPO) should be implemented $[27,28]$. Since duration of forward planning in this study was strongly correlated with the number of trajectories and dwell positions, it seemed feasible only for small lesions or lesions with favorable geometry (spherical). Similar to HDR or PSI, a workflow would start with CT-based target volume definition, dose prescription, and trajectory/dwell position calculation, followed by a suggestion of suitable trajectories. The puncture procedure would then consist of introducer needle or Kirschner wire and subsequent dilation, until a guidance tube could be placed to steer the needle applicator along the trajectory to the dwell positions. Strategies to move the needle applicator within the guidance tube already exist and include stereotactic frames or robotic guidance and can be combined with motion sensing and optical tracking $[29,30,31]$.

\section{Conclusions}

Interstitial irradiation of inoperable lesions with needle applicator-based stepping-source EBT allow for conformal PTV coverage with the prescribed doses. Adaption of the prescription dose utilizing an algorithm for $\mathrm{kV}$-photon distribution in heterogeneous tissue is feasible. Forward planning is achievable for small lesions but should be discarded in favor of inverse planning for larger PTV. The results of this planning study warrant the development of a clinical workflow.

\section{Acknowledgements}

We would like to gratefully acknowledge the assistance of Mr. Matthias Benker. Some parts of this work were presented at the ESTRO 38 Congress in Milan (EP-2157).

\section{Funding}

The underlying project was funded by DFG grants HE3011/36-1 and WE2063/11-1.

\section{Disclosure}

AMR, LI, JH, UO, and FW have nothing to disclose. FS, YAM, and FB report personal fees from CARL ZEISS MEDITEC AG, outside the submitted work. GRS reports grants and personal fees from CARL ZEISS MEDITEC AG, outside the submitted work. FG reports personal fees from Siemens Healthcare Diagnostics $\mathrm{GmbH}$, grants, and personal fees from NOXXON Pharma AG, grants and personal fees from CARL ZEISS MEDITEC AG, personal fees from Merck Serono $\mathrm{GmbH}$, personal fees from Roche Pharma AG, outside the submitted work; in addition, FG has a patent (US 62/435405) pending.

\section{References}

1. Hegde JV, Demanes DJ, Veruttipong D et al. Head and neck cancer reirradiation with interstitial high-dose-rate brachytherapy. Head Neck 2018; 40: 1524-1533.

2. Kolotas C, Röddiger S, Strassmann G et al. Palliative interstitial HDR brachytherapy for recurrent rectal cancer. Strahlenther Onkol 2003; 179: 458-463.

3. Han L, Li C, Wang J et al. Iodine-125 radioactive seed tissue implantation as a remedy treatment for recurrent cervical cancer. J Cancer Res Ther 2016; 12 (Supplement): C176-C180.

4. Yu YH, Wei CY, Qin QH et al. Efficacy of iodine-125 seed implantation in locoregionally recurrent and unresectable breast cancer: a retrospective study. Pathol Oncol Res 2019; 25: 327-332.

5. Guedea F, Venselaar J, Hoskin P et al. Patterns of care for brachytherapy in Europe: updated results. Radiother Oncol 2010; 97: 514-520.

6. Mobit PN, Packianathan S, He R et al. Comparison of Axxent-Xoft, (192)Ir and (60)Co high-dose-rate brachytherapy sources for image-guided brachytherapy treatment planning for cervical cancer. Br J Radiol 2015; 88: 20150010-20150010.

7. Schneider F, Clausen S, Jahnke A et al. Radiation protection for an intraoperative $\mathrm{X}$-ray source compared to C-arm fluoroscopy. Z Med Phys 2014; 24: 243-251.

8. Mobit PN, Rajaguru P, Brewer M et al. Radiation safety consideration during intraoperative radiation therapy. Radiat Prot Dosimetry 2015; 164: 376-382. 
9. Eaton DJ, Gonzalez R, Duck S et al. Radiation protection for an intra-operative X-ray device. Br J Radiol 2011; 84: 1034-1039.

10. Vaidya JS, Wenz F, Bulsara M et al. Risk-adapted targeted intraoperative radiotherapy versus whole-breast radiotherapy for breast cancer: 5 -year results for local control and overall survival from the TARGIT-A randomised trial. Lancet 2014; 383: 603-613.

11. Giordano FA, Brehmer S, Murle B et al. Intraoperative radiotherapy in newly diagnosed glioblastoma (INTRAGO): an open-label, dose-escalation phase I/II trial. Neurosurgery 2019; 84: 41-49.

12. Pantazis G, Trippel M, Birg W et al. Stereotactic interstitial radiosurgery with the photon radiosurgery system (PRS) for metastatic brain tumors: a prospective single-center clinical trial. Int J Radiat Oncol Biol Phys 2009; 75: 1392-1400.

13. Bludau F, Welzel G, Reis T et al. Combined kyphoplasty and intraoperative radiotherapy (Kypho-IORT) versus external beam radiotherapy (EBRT) for painful vertebral metastases a randomized phase III study. BMC Cancer 2019; 19: 430.

14. Schneider F, Fuchs H, Lorenz F et al. A novel device for intravaginal electronic brachytherapy. Int J Radiat Oncol Biol Phys 2009; 74: 1298-1305.

15. Bhatnagar A. Nonmelanoma skin cancer treated with electronic brachytherapy: results at 1 year. Brachytherapy 2013; 12: 134-140.

16. Lozares-Cordero S, Font-Gómez JA, Gandía-Martínez A et al. Treatment of cervical cancer with electronic brachytherapy. J Appl Clin Med Phys 2019; 20: 78-86.

17. Sethi A, Emami B, Small W, Jr. et al. Intraoperative radiotherapy with INTRABEAM: technical and dosimetric considerations. Front Oncol 2018; 8: 74.

18. Clausen S, Schneider F, Jahnke L et al. A Monte Carlo based source model for dose calculation of endovaginal TARGIT brachytherapy with INTRABEAM and a cylindrical applicator. Z Med Phys 2012; 22: 197-204.

19. Beaulieu L, Carlsson Tedgren A, Carrier JF et al. Report of the Task Group 186 on model-based dose calculation methods in brachytherapy beyond the TG-43 formalism: current status and recommendations for clinical implementation. Med Phys 2012; 39: 6208-6236.

20. van't Riet A, Mak ACA, Moerland MA et al. A conformation number to quantify the degree of conformality in brachytherapy and external beam irradiation: Application to the prostate. Int J Radiat Oncol Biol Phys 1997; 37: 731-736.

21. Baltas D, Kolotas C, Geramani K et al. A conformal index (COIN) to evaluate implant quality and dose specification in brachytherapy. Int J Radiat Oncol Biol Phys 1998; 40: 515-524.

22. van Leeuwen $\mathrm{CM}$, Oei AL, Crezee J et al. The alfa and beta of tumours: a review of parameters of the linear-quadratic model, derived from clinical radiotherapy studies. Radiat Oncol 2018; 13: 96.

23. Liu Q, Schneider F, Ma L et al. Relative biologic effectiveness (RBE) of $50 \mathrm{kV}$ X-rays measured in a phantom for intraoperative tumor-bed irradiation. Int J Radiat Oncol Biol Phys 2013; 85: 1127-1133.

24. Withers HR, Thames HD, Jr., Peters LJ. A new isoeffect curve for change in dose per fraction. Radiother Oncol 1983; 1: 187-191.

25. Eaton DJ. Electronic brachytherapy - current status and future directions. Br J Radiol 2015; 88: 20150002-20150002.

26. Serrano NA, Pham HT, Narayanan S et al. Permanent prostate brachytherapy using high V150. Pract Radiat Oncol 2015; 5: e201-206.

27. Trnkova P, Baltas D, Karabis A et al. A detailed dosimetric comparison between manual and inverse plans in HDR intracavitary/interstitial cervical cancer brachytherapy. J Contemp Brachytherapy 2010; 2: 163-170.
28. Cunha JA, Hsu IC, Pouliot J et al. Toward adaptive stereotactic robotic brachytherapy for prostate cancer: demonstration of an adaptive workflow incorporating inverse planning and an MR stealth robot. Minim Invasive Ther Allied Technol 2010; 19: 189-202.

29. Maier-Hein L, Tekbas A, Seitel A et al. In vivo accuracy assessment of a needle-based navigation system for CT-guided radiofrequency ablation of the liver. Med Phys 2008; 35 : 5385-5396.

30. Brehmer S, Guthier CV, Clausen S et al. Combined stereotactic biopsy and stepping-source interstitial irradiation of glioblastoma multiforme. J Neurosurg Sci 2018; 62: 214-220.

31. M2OLIE: Robotic Kypho IORT (RoRaTh/UMM). Available at: https:/ / www.youtube.com/watch?v=pmdsdBC63uI (accessed 20 January 2020). 